\title{
Comparison of Blue and White Lamp Light with Sunlight for Daylight-Mediated, 5-ALA Photodynamic Therapy, in vivo
}

\author{
Kayla Marra ${ }^{*}, 1$, Ethan P. LaRochelle ${ }^{1}$, M. Shane Chapman ${ }^{2}$, P. Jack Hoopes ${ }^{2}$, Karina \\ Lukovits ${ }^{1}$, Edward V. Maytin ${ }^{3}$, Tayyaba Hasan ${ }^{4}$, and Brian W. Pogue ${ }^{1,2}$ \\ ${ }^{1}$ Thayer School of Engineering, Dartmouth College, Hanover NH 03755 \\ 2Department of Surgery, Geisel School of Medicine, Hanover NH 03755 \\ ${ }^{3}$ Department of Biomedical Engineering, Learner Research Institute, Cleveland Clinic Foundation, \\ Cleveland $\mathrm{OH} 44195$ \\ ${ }^{4}$ Wellman Center for Photomedicine, Massachusetts General Hospital, Harvard Medical School, \\ Boston MA 02114
}

\begin{abstract}
Daylight-mediated photodynamic therapy (d-PDT) as a treatment for actinic keratosis (AK) is an increasingly common technique due to a significant reduction in pain, leading to better patient tolerability. While past studies have looked at different light sources and delivery methods, this study strives to provide equivalent PpIX-weighted light doses with the hypothesis that artificial light sources could be equally as effective as natural sunlight if their PpIX-weighted fluences were equalized. Normal mouse skin was used as the model to compare blue LED light, metal halide white light, and natural sunlight, with minimal incubation time between topical ALA application and the onset of light delivery. A total PpIX-weighted fluence of $20 \mathrm{~J}_{\mathrm{eff}} / \mathrm{cm}^{2}$ was delivered over 2 hours, and the efficacy of response was quantified using three acute bioassays for PDT damage: PpIX photobleaching, Stat 3 crosslinking, and quantitative histopathology. These bioassays indicated blue light was slightly inferior to both sunlight and white light, but that the latter two were not significantly different. The results suggest that metal halide white light could be a reasonable alternative to daylight PDT, which should allow a more controlled treatment that is independent of weather and yet should have similar response rates with limited pain during treatment.
\end{abstract}

\section{Graphical abstract}

In this study three light sources were directly compared for daylight-mediated photodynamic therapy efficacy. Two artificial light sources, a blue LED and a ceramic metal halide white light were compared to natural sunlight. Each source's spectral irradiance was normalized to the absorption spectrum of the photosensitizer, protoporphyrin IX (PpIX), to deliver a precisely matched total light dose. Acute bioassays including immunoblot and quantitative histopathology,

\footnotetext{
*Corresponding author's: kayla.marra@ dartmouth.edu (Kayla Marra).

Supporting Information: Additional Supporting Information can be found in the online version of this article:
} 
pictured above, reveal that all three light sources were effective mediators, though blue light was found to be slightly inferior.

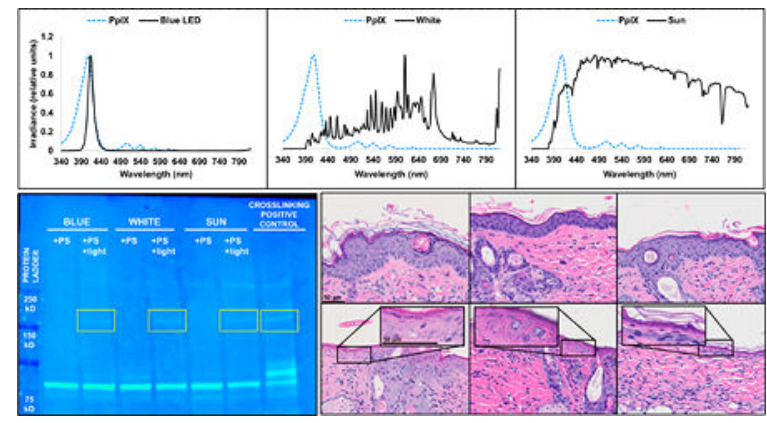

\section{Introduction}

Photodynamic therapy (PDT) is a treatment strategy that combines a light-sensitive drug called a photosensitizer (PS), with excitation light of the appropriate wavelength(s), and through collisional quenching by tissue oxygen, generates cytotoxic molecules that can induce necrosis and apoptosis of tissues in a spatially controlled manner (1-3). PDT has become a widely utilized option for superficial, dermatological conditions, with superior cosmetic results (4). In the U.S. it is approved to treat actinic keratosis (AK), and in Europe it is approved for AK, Bowen's Disease, superficial basal cell carcinomas (sBCC), and in some cases, thin nodular BCCs (5). There has also been expanding interest in anti-microbial applications for acne vulgaris (6-8), as well as the cosmetic field of photorejuvenation $(9,10)$. As PDT has become more widely practiced in clinics for these indications, translational clinical researchers have sought to optimize patient compliance and satisfaction, with a focus on pain reduction. PDT-associated pain varies from patient to patient, but it can sometimes prove intolerable (11-13). In response to this, Wiegell et al. demonstrated that the combination of low-intensity light and the significant reduction of the PS incubation time can together greatly reduce the patient's perception of pain, without sacrificing efficacy for the treatment of AK (14). Since then, daylight mediated PDT (dPDT) has become internationally recognized as an effective treatment strategy for AK (1519). In addition to high tolerability, d-PDT has added benefits such as the ability to treat a large area of multiple lesions simultaneously, improved clinical workflow due to the removal of the drug incubation step, and the ability to treat multiple patients simultaneously.

The innovation of natural sunlight as a mediator for PDT has introduced the need for standard methods of reporting and comparing light doses, which can vary in spectrum or fluence rate. While there has been some inconsistency in the literature about reporting the spectral irradiance of the sun in a manner that allows comparison between research groups, the initial Wiegell paper suggested that disparate light sources could be directly compared by normalizing their raw output to the absorption spectrum of protoporphyrin IX (PpIX) (14). In practice, this has been done by most reports now that use d-PDT, and this method was adopted here to allow for a quantitative comparison between different lamps and light sources. While this approach solves a large fraction of the inter-light source comparisons, it should also be noted that the exact PpIX spectrum used can vary depending upon the 
solvent, and in this study we chose PpIX dimethyl ester dissolved in chloroform (20).

Additionally, the effects of tissue optical properties are not accounted for in this approach, but this will be discussed after the results of this paper.

The question tested in this study was, could two artificial light sources, blue LED and metal halide white light, be as equally effective as sunlight if the delivered fluences are equal when normalized to PpIX? This study evaluated the biological response to PDT by the chosen light sources via three acute indicators of PDT damage, as we have previously described (21). Briefly, PpIX photobleaching, Stat 3 crosslinking, and traditional histopathology were used as in situ measures of physical dosimetry, biochemical damage and biological damage, respectively. Normal nude mouse skin was used as the model to investigate these questions, because of its exquisite homogeneous production of PpIX $(22,23)$. The entering hypothesis was that although the spectral signatures between the three light sources were different, careful normalization to PpIX's absorption spectrum would yield equally effective results.

\section{Materials and Methods}

\section{Light Sources}

For the purposes of this study we wanted to choose three distinct light sources that would have generous spectral overlap with PpIX's absorption spectrum and would be able to deliver $20 \mathrm{~J}$ eff $/ \mathrm{cm}^{2}$ over approximately 2 hours. The selected lights were a $415 \mathrm{~nm}$ LED (Light Therapy Options, Thomaston, CT) a broad spectrum, ceramic metal halide (Philips, Amsterdam, Netherlands) and natural sunlight (fig. 1). It should be noted that for simplicity, we will refer to these lights as blue, white, and sun, respectively, but with regard to the metal halide in particular the spectrum is very specific to the type of bulb used. To help filter harmful UV wavelengths, a consideration necessary for human translation, an acrylic glass sheet was placed between the light source and mouse during spectral measurements and treatment (Supporting Information, fig. S1). Spectral data were captured using the SS-110 Field Spectroradiometer (Apogee Instruments, Logan, UT). The instrument was last calibrated April 2017, prior to any data collection, and is valid until April 2019. Calibration was performed by the manufacturer with a LI-COR model 1800-02 Optical Radiation Calibrator using a 200 Watt quartz halogen lamp. The 1800-02 and quartz halogen lamp are traceable to the National Institute of Standards and Technology (NIST). Using the spectroradiometer, we verified that the irradiance of each light source would be uniform across the treatment field (approximately $50 \mathrm{~mm} \times 38 \mathrm{~mm}$, data not shown).

\section{PpIX Weighting}

For dose planning and verification, the emission spectrum of each light source was weighted to the absorption spectrum of PpIX. For a given PpIX spectrum, the peak maximum was determined and assigned a value of 1 . All other wavelengths were expressed as a fraction of the peak maximum (0-1). We then multiplied this normalized spectrum by the absolute spectral irradiance of each light source to determine the PpIX-weighted irradiance. We aimed to deliver $20 \mathrm{~J}_{\mathrm{eff}} / \mathrm{cm}^{2}$ over approximately $120 \mathrm{mins}$. For the artificial light sources (blue \& white), spectral measurements were taken pre-treatment and the distance from light to animal was adjusted to deliver approximately $2.78 \mathrm{~mW}_{\mathrm{eff}} / \mathrm{cm}^{2}$. For natural sunlight, we 
took real-time spectral measurements (one per minute) and calculated the appropriate stopping time.

\section{Photodynamic Therapy in vivo}

For these studies normal nude mouse skin was used as the model. All animal procedures were carried out in accordance with Dartmouth's Institutional Animal Care and Use Committee. 30 female mice ( 20 weeks old, Charles River Laboratories, Wilmington, MA) were divided evenly among the three light groups. The treatment field was divided into 4 equal areas, $9.5 \mathrm{~mm} \times 12.5 \mathrm{~mm}$ each, for a total field of $50 \mathrm{~mm} \times 38 \mathrm{~mm}$ ([1 control +1 treated] $\times 2$ assays $=4$, fig. 2). Prior to treatment, baseline PpIX fluorescence measurements were taken using a dual channel fiber optic probe, as previously described $(21,24)$. After measurements, Levulan Kerastick ${ }^{\circledR}$ (20\% 5-ALA) was applied evenly to each area. Each animal served as its own control, so half of the field was occluded with light-impermeable dressing (control $=+\mathrm{PS}$, - light, fig. $2 \mathrm{~b}-\mathrm{c}$ ). To prevent potential anatomical artifact, the occluded field was randomized to either the upper or lower back for every other animal, such that each light group had a total of 5 upper and 5 lower control fields. Immediately after the pro-drug was applied and the dressing was affixed ( $\sim 5 \mathrm{~min})$ the animal was exposed to light for approximately 2 hours. Over the course of treatment, internal body temperature was continuously monitored by a rectal thermometer (Kent Scientific, Torrington, CT) and maintained within $\pm 0.5^{\circ} \mathrm{C}$ of $37^{\circ} \mathrm{C}$. This was done by placing a gentle desk fan in front of the mice receiving white light or sun; the blue light group required a heating pad. Upon completion of the light dose, post-treatment PpIX measurements were taken and two skin biopsies were harvested (one treated, one control, fig. 2c) for Stat3 analysis. After 24 hours post treatment, the animal was sacrificed and the two remaining skin biopsies were taken for H\&E staining (one treated, one control, fig. 2c).

\section{Protein isolation \& quantification for Stat3 analysis}

Biopsies approximately $9.5 \mathrm{~mm} \times 12.5 \mathrm{~mm}$ in size were taken from the treatment plane and immediately placed on ice in $\sim 800 \mu$ RIPA buffer (Sigma-Aldrich, St. Louis, MO) containing $1 \mathrm{mM}$ PMSF, protease (Sigma-Aldrich) and phosphatase inhibitors (Thermo Fisher Scientific, Waltham, MA). Samples were allowed to equilibrate on ice for 20 minutes before physical homogenization, followed by centrifugation for 5 minutes at max speed. The supernatant was then carefully transferred to a clean vial for storage at $-20^{\circ} \mathrm{C}$ until ready for use. Protein concentrations were quantified using BCA assay (Thermo Fisher).

\section{Quantitative Western Blotting}

Stat 3 crosslinking was interrogated by SDS-PAGE and immunoblotting, as previously described (21). Briefly, samples containing $50 \mu \mathrm{g}$ protein were denatured and separated on a 4-15\% polyacrylamide gel (Bio-Rad Laboratories, Hercules, CA). Proteins were then transferred to a $0.2 \mu \mathrm{m}$ PVDF membrane (Bio-Rad) using the Bio-Rad Trans-Blot Turbo transfer system. To block non-specific interactions the membrane was incubated with 5\% powdered skim milk in PBS for 1 hour at room temperature. Primary antibody staining occurred overnight at $4^{\circ} \mathrm{C}$, using the Santa Cruz (Dallas, TX) Stat3 antibody (F-2), at a dilution of 1:200 in a 5\% BSA, PBST solution. The next day, the membrane was washed $3 \times$ for $5 \mathrm{~min}$ in PBST and incubated with a fluorescently labeled secondary antibody (IRDye ${ }^{\circledR}$ 
800CW Goat anti-Mouse IgG, LI-COR Biosciences, Lincoln, NE) at a dilution of 1:15,000 for one hour at room temp. After a final set of three washes, the membrane was imaged using the LI-COR Odyssey CLx. Crosslinking levels were normalized to monomer and band intensities were quantified using the LI-COR Image Studio ${ }^{\text {TMLite }}$ software.

\section{H\&E analysis - nuclei counting}

To quantify epidermal keratinocyte damage, we counted the number of healthy or normal appearing nuclei in the epidermis, as previously described (21). Slides were scanned on the Vectra automated slide scanner (Perkin Elmer, Waltham, MA) at 40× resolution $(334 \times 240$ $\mu \mathrm{m}^{2}$ ). Five regions of interest were randomly scanned per biopsy (a total area of $1667 \times$ $1200 \mu^{2}$ ). Using Fiji (25), regions of interest (ROIs) were drawn around the epidermis. We then used the IHC Toolbox plugin to segment and count nuclei $(26,27)$. The algorithm could be visually verified by an overlay that was produced demarcating the counted nuclei. However, we found that the algorithm was only successful about $50 \%$ of the time, perhaps due to inherent differences in the staining contrast. The images that "failed" were then manually counted. For each biopsy, the five ROIs were summed for a total count.

\section{H\&E analysis - hematoxylin histograms}

The same five regions of interest per biopsy described above were analyzed here (a total area of $1667 \times 1200 \mu \mathrm{m}^{2}$ ). Again, using Fiji, the same ROIs were selected around the epidermis. We then used a color deconvolution plugin $(28,29)$ to separate the composite image into its individual hematoxylin and eosin components. From here, a pixel intensity histogram was generated of the hematoxylin stained image (within the epidermis). For each biopsy, the five histograms were summed. Histograms were then smoothed using a moving median filter, and the peak maxima were compared for statistical evaluation.

\section{Statistical Evaluation}

Intragroup analyses (control v. treated) were performed using a two-tailed, paired Student's $\mathrm{T}$ Test. Intergroup analyses were performed with a two-tailed, two sample, assumed unequal variance Student's T Test. For all boxplots, the mean is indicated by a dotted line. The box represents the interquartile range and the whiskers represent the max and min. If outliers are present, they are displayed as filled circles. Only significant p-values $(<0.05)$ are given above the corresponding data.

\section{Results}

\section{Small changes in PpIX's absorption spectrum affect the calculated weighted light dose}

Not surprisingly, small solvent and/or concentration shifts in PpIX's absorption spectrum can cause significant differences when calculating the cumulative weighted light dose. We compared a spectrum that has been widely used for dose-weighting in the literature (30-32) (Peng, fig. 3) to a standard appearing in the PhotochemCAD library (20) (Taniguchi, fig. 3) that we have used in the current and previous study (21). Additionally, we collected our own spectrum to serve as a reality check (Marra, fig. 3). We retrospectively calculated a total fluence using each spectrum (table 1), but for actual dose planning during this study, we standardized our light sources to the Taniguchi spectrum. 


\section{Blue, white, \& sun are effective in their ability to photobleach PpIX}

PpIX levels can vary widely from patient to patient, and have been shown to correlate to patient pain as well as clearance outcome $(33,34)$. Therefore, it is good standard practice to monitor PpIX production and photobleaching to correlate to results. Typically, PpIX levels are measured after the incubation of 5-ALA (or its methyl ester) for several hours to assess the buildup of the photosensitizer within the lesion. However, in the d-PDT modality there is little or no incubation of the pro-drug and therefore no PpIX accumulation. As such, we had to infer PpIX accumulation from the covered control field. We saw that in each light group there was significant build-up under the occlusion, while only nominal levels of PpIX could be detected in the light-exposed treatment field (fig. 4). The PpIX fluorescence dosimeter is equipped with two different excitation lasers, blue and red. By blue laser excitation (fig. 4a), we can see that PpIX values had accumulated in the control tissue to $1.4 \pm 0.5,0.7 \pm 0.4$, and $0.7 \pm 0.2$ for blue, white, and sun, respectively (a.u., mean $\pm \mathrm{SD}$ ). In contrast, the treated tissue was successfully photobleached to $0.1 \pm 0.1,0.1 \pm 0.0$, and $0.2 \pm 0.2$ for blue, white, and sun respectively. This indicates that each light source was effective in activating the PpIX that was available. Likewise, red laser excitation (fig. 4b) shows high levels of PpIX accumulation in the control tissue $(2.2 \pm 1.0,1.0 \pm 0.6$, and $1.3 \pm 0.6$; blue, white, sun) and minimal levels in the treated tissue $(0.1 \pm 0.3,0.3 \pm 0.3$, and $0.3 \pm 0.4$; blue, white, sun). Interestingly, we observed that the white $\&$ sun groups had half as much build-up of PpIX in their control fields than the blue group $(\mathrm{p}<0.05)$.

\section{Blue, white, \& sun induce Stat3 crosslinking}

Stat 3 crosslinking has been demonstrated in the literature to be an immediate indicator of PDT efficacy and is dosimetric in nature, with regard to light dose and photosensitizer concentration $(21,35,36)$. Figure 5 shows that there is a significant increase in Stat3 crosslinking (expressed as a percentage of monomer) in all three light groups, with mean values of $3.3 \pm 1.7,6.1 \pm 2.6$, and $4.6 \pm 3.0$ for blue, white, and sun, respectively ( $\pm \mathrm{SD}$ ). This was compared to control tissue where crosslinking values were $0.4 \pm 0.5,0.6 \pm 0.7$, and $0.7 \pm 0.6$ (blue, white, sun) and could not be detected by eye on the immunoblot (Fig 5a). We can also observe that there was significantly less crosslinking, by a factor of 2 , in the blue group than in the white group ( $\mathrm{p}<0.05)$.

\section{Histopathology shows cellular damage in each light group, mainly localized to the epidermis}

At 24 hours post-treatment we see significant keratinocyte damage localized to the epidermis (fig. 6). When we quantified this damage, we noticed a significant difference between the treated and control tissue in each light group (fig. 7). The mean number of healthy nuclei $( \pm$ SD) visible in the epidermal layer of the control tissue were $457 \pm 106$, $483 \pm 105$, and $\pm 448 \pm 119$ for blue, white, and sun, respectively. This was decreased in the treated tissue to $301 \pm 193,200 \pm 87$, and $134 \pm 85$ for blue, white, and sun, respectively. Additionally, we can see that there was a 2 -fold difference between the damage inflicted by sun compared to the damage inflicted by blue light $(\mathrm{p}<0.05)$.

Because of the low accuracy that often accompanies segmenting algorithms, and the burden of manually counting thousands of nuclei, we sought to determine a simpler and more time- 
efficient method to quantify epidermal damage. Cellular damage is characteristically distinguished by a shrinking of the nuclei, as well as hyper-eosinophilia (an increase in the pink staining, indicative of cellular membrane breakdown that exposes proteins to this dye). We therefore posited that damage would be correlated to a loss of purple staining. Hematoxylin histograms indicate that there is a decrease in the area of the epidermis (shrinkage) and that there is a shift in the intensity of staining (fig. 8). These changes can be quantified by a shift in the peak maximum. When analyzed, we see very similar patterns to the counting method, and an $\mathrm{r}^{2}$ value of 0.74 (fig. 9), suggesting this method could be used to rapidly and accurately quantify epidermal damage.

\section{Discussion}

In the present study, the question posed was, could two different artificial light sources be equally as effective as sunlight if the delivered light doses are carefully matched to PpIX's absorption spectrum? The goal was to assess if blue light treatment could be utilized in a low irradiance and low incubation time approach, thereby leading to lower pain in clinical treatments, similar to what is reported by d-PDT. The metal halide lamp was chosen to mimic natural sunlight, but is readily accessible and controlled in a clinic. Our treatment protocol deviated slightly from what is commonly practiced in Europe and Australia, because there the methyl ester of ALA (MAL) is used, which is formulated in a white opaque cream. In that application, irradiation is done after 30 minutes, partly because the MAL must be removed before light treatment can commence usefully. This is why it is typically allowed to incubate for 30 minutes prior to light exposure in the d-PDT modality. The Levulan that is approved in the US and was used in this study is a clear formulation and therefore no removal step is needed. So one potential advantage of Levulan over MAL is this ability to have no waiting period between ALA administration and irradiation.

In a previous study from our group (21), a comparison between the efficacies of lowintensity artificial and natural sunlight to conventional \& fractionated PDT was initially attempted. The results demonstrated that a variety of acute biomarkers that are dosimetric in nature could be used in future studies to rapidly quantify PDT efficacy. The current study employed these assays and in summary, the results confirm that each light source was effective across the three acute indicators used to assess PDT efficacy: i) the capacity to photobleach PpIX, ii) the induction of Stat 3 crosslinking, and iii) the induction of observable histopathological damage to epidermal nuclei (table 2). However, as the results also indicate, the blue light treatment group was slightly less effective than sun and white light.

Stat3 and histopathology analyses together reveal that blue light is a somewhat inferior mediator of PDT. With regard to Stat 3 crosslinking, it was observed that blue light was less effective than white by a factor of $2(\mathrm{p}<0.05)$, but that sunlight was not significantly different from either group. This is complementary to the H\&E data, where blue light irradiation was inferior to sunlight by a factor of $2(\mathrm{p}<0.05)$, while white light was not significantly different. Taken together, it is possible to conclude that blue was inferior to both lights, while white lamp and sun are equally effective mediators. This conclusion challenges the current hypothesis that all lights would perform equally when weighted to the absorption spectrum of PpIX. 
One theory for the less favorable effect from blue light could be the limited depth of tissue penetration at these wavelengths. Valentine et al. found through Monte Carlo simulations that $405 \mathrm{~nm}$ light could necrose tumors up to a depth of $2.23 \mathrm{~mm}$ and $620 \mathrm{~nm}$ light could necrose to depths of $3.81 \mathrm{~mm}$ (37), all else being equal. However, in the current study, no observations of any depth differences were seen in the pathology data, which showed that damage was mostly confined to the epidermis and the hair follicles, with an average depth of $\sim 60 \mu \mathrm{m}$ (Supporting Information, fig. S2). This is much shallower than the expected light depth penetration reported in the literature. A possible explanation is that the limiting factor here is not light penetration, but the diffusion rate of the 5-ALA. This could be especially true for daylight PDT protocols, where there is no protected incubation time of the photosensitizer, but rather the pro-drug and light administered simultaneously. It seems obvious that with longer incubation times 5-ALA, and possibly PpIX, would diffuse deeper into the skin, and in this situation one would expect to see the wavelength-dependent penetration exert a greater effect. Juzenas et al. experimentally determined the diffusion coefficient for 5-ALA to be $0.16+/-0.03 \mathrm{~mm}^{2}$ per hour (38). In another Monte Carlo paper, Campbell et al. demonstrated that daylight can treat tumors at depths of $2 \mathrm{~mm}$, but this model assumed that PpIX had already accumulated at depth (39). When their model decreased PpIX's concentration by a factor of three, they obtained $\sim 1 \mathrm{~mm}$ depth damage. This is still $\sim 10$ fold greater than the depths that we observed, but a key difference is that our study employed normal mouse skin. Studies have shown that PpIX photosensitization rarely occurs in the dermis (40), and so the question of depth efficacy is likely beyond the scope of this study. Still, it would be interesting to determine the exact depth-to-time ratio, and perhaps incubation times could be finessed to treat thicker lesions, with the understanding that there will most likely be a trade-off with the patient's perception of pain.

The overall attenuation by tissue optics from both absorption and scattering, does dramatically decrease the available light with increasing wavelength $(41,42)$. Without accounting for this factor, the calculated PpIX-effective light dose actually overestimates the dose delivered by the blue light. It would follow that the broader band light sources, white and sun, could actually be delivering a stronger effective dose, and in the future a more accurate weighting factor could be a combination of PpIX's absorption spectrum modified by the penetration by the tissue optical properties down to the depth of tissue where PpIX production is important. To complicate matters, small shifts in PpIX's absorption spectrum can exacerbate discrepancies in the effective light dose, and it makes cross-study comparisons difficult. For example, Rubel et al. found that they were unable to achieve a comparable sunlight dose by a factor of two, stating "Moreover, the effective light dose we observed $\left(22.8 \mathrm{~J} \mathrm{~cm}^{2}\right)$ was lower than that observed by Wiegell et al. $\left(43.2 \mathrm{~J} \mathrm{~cm}^{2}\right)$, perhaps due to the fact that their study was performed in the summer" $(14,43)$. Currently, it is not easy to determine what the true, physiological absorption spectrum of PpIX looks like in vivo, and our study is limited in that it was done in pre-clinical mouse skin. Future studies quoting weighted PpIX fluence values should ideally reference or publish the exact PpIX absorption spectrum that was used for calculating the effective light dose, so that cross-study comparisons may be more standardized.

Finally, we observed that there was a slight response difference between the groups with regard to the post-treatment PpIX fluorescence measurements. Figure 4 shows that there was 
nearly a two-fold increase in PpIX accumulation in the blue group's control field ( $\mathrm{p}<0.05)$. We do not have a good explanation for this. We are confident that the white light and sunlight were not able to directly penetrate the occlusive dressing, which was tested by covering the spectroradiometer with the material and taking measurements (Supporting Information, fig. S3). There should not have been any biological differences among the animals in the different light groups. We made efforts to maintain core body temperature, but it could be that the surface temperature was more variable as this was not directly measured, and it is well documented that temperature affects PDT efficacy $(44,45)$. It could be possible that the longer wavelengths present in the white light and sun are scattered within the tissue, and photons may be able to travel farther and perhaps reach the control field, but this was not tested. At any rate, we did not observe any significant damage in the control histopathology, so although this is an interesting observation it is not impactful to this study.

In this study, the efficacies of two different low irradiance artificial light sources were compared to sunlight based PDT, with the hypothesis that exact dose matching to PpIX's absorption spectrum would yield equal results. Using a normal mouse skin model, with minimal time delay between ALA application and onset of light delivery, the results show that the blue light group was slightly inferior to both artificial metal halide white light and natural sunlight, as quantified by three acute biomarkers of PDT efficacy: PpIX photobleaching, Stat 3 crosslinking, and histopathology. The observed differences are hypothesized to be from wavelength dependent light attenuation of exclusively blue light, relative to the broader spectrum metal halide white light or natural sunlight, by the biological tissue optical properties. However, the efficacy of the artificial metal halide appears to be identical to natural sunlight in this murine skin model, when delivered under these controlled conditions. These data suggest that daylight PDT might be attempted with metal halide lamp irradiation and that a very similar control of lesions could be expected, compared to sun. The added value is that this treatment would be independent of uncontrolled factors such as weather, and it would allow this painless PDT approach to be used in more northern climates or in less reliable weather patterns, without sacrificing efficacy.

\section{Supplementary Material}

Refer to Web version on PubMed Central for supplementary material.

\section{Acknowledgments}

This work has been funded by NIH research grant P01 CA84203, as well as the Norris Cotton Cancer Center preclinical imaging and microscopy shared resource P30 CA023108.

\section{References}

1. Kelly JF, Snell ME. Hematoporphyrin derivative: a possible aid in the diagnosis and therapy of carcinoma of the bladder. J Urol. 1976; 115:150-1. [PubMed: 1249866]

2. Dougherty TJ, et al. Photoradiation therapy for the treatment of malignant tumors. Cancer Res. 1978; 38:2628-35. [PubMed: 667856]

3. Henderson BW, Dougherty TJ. How Does Photodynamic Therapy Work? Photochem Photobiol. 1992; 55:145-157. [PubMed: 1603846] 
4. Griffin LL, Lear JT, Photodynamic Therapy. Non-Melanoma Skin Cancer. Cancers (Basel). 2016; 8

5. Morton CA, McKenna KE, Rhodes LE. Guidelines for topical photodynamic therapy: update. Br J Dermatol. 2008; 159:1245-1266. [PubMed: 18945319]

6. Hongcharu W, et al. Topical ALA-Photodynamic Therapy for the Treatment of Acne Vulgaris. J Invest Dermatol. 2000; 115:183-192. [PubMed: 10951234]

7. Itoh Y, Ninomiya Y, Tajima S, Ishibashi A. Photodynamic Therapy for Acne Vulgaris With Topical 5-Aminolevulinic Acid. Arch Dermatol. 2000; 136:1093-1095. [PubMed: 10987863]

8. Chen X, et al. Clinical efficacy of 5-aminolevulinic acid photodynamic therapy in the treatment of moderate to severe facial acne vulgaris. Exp Ther Med. 2015; 10:1194-1198. [PubMed: 26622463]

9. Dover JS, Bhatia AC, Stewart B, Arndt KA. Topical 5-Aminolevulinic Acid Combined With Intense Pulsed Light in the Treatment of Photoaging. Arch Dermatol. 2005; 141:1247-1252. [PubMed: 16230562]

10. Jang YH, Koo GB, Kim JY, Kim YS, Kim YC. Prolonged Activation of ERK Contributes to the Photorejuvenation Effect in Photodynamic Therapy in Human Dermal Fibroblasts. J Invest Dermatol. 2013; 133:2265-2275. [PubMed: 23337889]

11. Miller IM, Nielsen JS, Lophaven S, Jemec GBE. Factors related to pain during routine photodynamic therapy: a descriptive study of 301 patients. J Eur Acad Dermatology Venereol. 2011; 25:1275-1281.

12. Dixon AJ, Anderson SJ, Dixon MP, Dixon JB. Post procedural pain with photodynamic therapy is more severe than skin surgery. J Plast Reconstr Aesthetic Surg. 2015; 68:e28-e32.

13. Wang B, et al. Gain with no pain? Pain management in dermatological photodynamic therapy. Br J Dermatol. 2017; doi: 10.1111/bjd.15344

14. Wiegell SR, et al. Continuous activation of PpIX by daylight is as effective as and less painful than conventional photodynamic therapy for actinic keratoses; a randomized, controlled, single-blinded study. Br J Dermatol. 2008; 158:740-746. [PubMed: 18294318]

15. Morton CA, et al. Practical approach to the use of daylight photodynamic therapy with topical methyl aminolevulinate for actinic keratosis: a European consensus. J Eur Acad Dermatology Venereol. 2015; 29:1718-1723.

16. Gilaberte Y, et al. Spanish-Portuguese consensus statement on use of daylight-mediated photodynamic therapy with methyl aminolevulinate in the treatment of actinic keratosis. Actas Dermosifiliogr. 2015; 106:623-631. [PubMed: 26115793]

17. Grinblat B, et al. Daylight-mediated photodynamic therapy for actinic damage in Latin America: consensus recommendations. Photodermatol Photoimmunol Photomed. 2016; 32:81-87. [PubMed: 26541910]

18. See JA, et al. Consensus recommendations on the use of daylight photodynamic therapy with methyl aminolevulinate cream for actinic keratoses in Australia. Australas J Dermatol. 2016; 57:167-174. [PubMed: 26033230]

19. Ibbotson $\mathrm{S}$, et al. A consensus on the use of daylight photodynamic therapy in the UK. J Dermatolog Treat. 2016; :1-8. DOI: 10.1080/09546634.2016.1240863 [PubMed: 26651369]

20. Taniguchi M, Lindsey JS. Database of Absorption and Fluorescence Spectra of $>300$ Common Compounds for use in PhotochemCAD. Photochem Photobiol. 2018; 94:290-327. [PubMed: 29166537]

21. de Souza ALR, et al. Assessing daylight \& low-dose rate photodynamic therapy efficacy, using biomarkers of photophysical, biochemical and biological damage metrics in situ. Photodiagnosis Photodyn Ther. 2017; 20:227-233. [PubMed: 29037911]

22. Robinson DJ, et al. Fluorescence photobleaching of ALA-induced protoporphyrin IX during photodynamic therapy of normal hairless mouse skin: the effect of light dose and irradiance and the resulting biological effect. Photochem Photobiol. 1998; 67:140-9. [PubMed: 9477772]

23. Juzeniene A, Juzenas P, Iani V, Moan J. Topical application of 5-aminolevulinic acid and its methylester, hexylester and octylester derivatives: considerations for dosimetry in mouse skin model. Photochem Photobiol. 2002; 76:329-34. [PubMed: 12403455]

24. Kanick SC, et al. Dual-channel red/blue fluorescence dosimetry with broadband reflectance spectroscopic correction measures protoporphyrin IX production during photodynamic therapy of actinic keratosis. J Biomed Opt. 2014; 19:75002. [PubMed: 24996661] 
25. Schindelin J, et al. Fiji: an open-source platform for biological-image analysis. Nat Methods. 2012; 9:676-682. [PubMed: 22743772]

26. Shu J, Guoping Qiu G. Immunohistochemistry (IHC) Image Analysis Toolbox. imagej.nih.gov. 2015. Available at: https://imagej.nih.gov/ij/plugins/ihc-toolbox/index.htmlAccessed: 21 st February 2018

27. Jie Shu J, Hao Fu H, Guoping Qiu G, Kaye P, Ilyas M. 2013 35th Annual International Conference of the IEEE Engineering in Medicine and Biology Society (EMBC). IEEE; 2013. Segmenting overlapping cell nuclei in digital histopathology images; 5445-5448. 2013

28. Landini G. [Accessed: 21st February 2018] Colour Deconvolution. imagej.net. 2017. Available at: https://imagej.net/Colour_Deconvolution

29. Ruifrok AC, Johnston DA. Quantification of histochemical staining by color deconvolution. Anal Quant Cytol Histol. 2001; 23:291-9. [PubMed: 11531144]

30. Peng Q, et al. 5-Aminolevulinic acid-based photodynamic therapy. Cancer. 1997; 79:2282-2308. [PubMed: 9191516]

31. Wiegell SR, Haedersdal M, Eriksen P, Wulf HC. Photodynamic therapy of actinic keratoses with $8 \%$ and $16 \%$ methyl aminolaevulinate and home-based daylight exposure: a double-blinded randomized clinical trial. Br J Dermatol. 2009; 160:1308-1314. [PubMed: 19416257]

32. Lerche C, Heerfordt I, Heydenreich J, Wulf H. Alternatives to Outdoor Daylight Illumination for Photodynamic Therapy-Use of Greenhouses and Artificial Light Sources. Int J Mol Sci. 2016; 17:309. [PubMed: 26938525]

33. Piffaretti F, et al. Correlation between Protoporphyrin IX Fluorescence Intensity, Photobleaching, Pain and Clinical Outcome of Actinic Keratosis Treated by Photodynamic Therapy. Dermatology. 2013; 227:214-225. [PubMed: 24135436]

34. Kanick S, et al. Pre-treatment protoporphyrin IX concentration in actinic keratosis lesions may be a predictive biomarker of response to aminolevulinic-acid based photodynamic therapy. Photodiagnosis Photodyn Ther. 2015; 12:561-566. [PubMed: 26480810]

35. Liu W, Oseroff AR, Baumann H. Photodynamic Therapy Causes Cross-linking of Signal Transducer and Activator of Transcription Proteins and Attenuation of Interleukin-6 Cytokine Responsiveness in Epithelial Cells. CANCER Res. 2004; 64:6579-6587. [PubMed: 15374971]

36. Henderson BW, et al. Cross-linking of signal transducer and activator of transcription 3 - A molecular marker for the photodynamic reaction in cells and tumors. Clin Cancer Res. 2007; 13:3156-3163. [PubMed: 17545518]

37. Valentine RM, Wood K, Brown CTA, Ibbotson SH, Moseley H. Monte Carlo simulations for optimal light delivery in photodynamic therapy of non-melanoma skin cancer. Phys Med Biol. 2012; 57:6327-6345. [PubMed: 22990348]

38. Juzenas P, Juzeniene A, Iani V, Moan J. Depth Profile of Protoporphyrin IX Fluorescence in an Amelanotic Mouse Melanoma Model. Photochem Photobiol. 2009; 85:760-764. [PubMed: 19140894]

39. Campbell CL, Wood K, Valentine RM, Brown CTA, Moseley H. Monte Carlo modelling of daylight activated photodynamic therapy. Phys Med Biol. 2015; 60:4059-4073. [PubMed: 25927971]

40. Divaris DX, Kennedy JC, Pottier RH. Phototoxic damage to sebaceous glands and hair follicles of mice after systemic administration of 5-aminolevulinic acid correlates with localized protoporphyrin IX fluorescence. Am J Pathol. 1990; 136:891-7. [PubMed: 2327473]

41. Lister T, Wright PA, Chappell PH. Optical properties of human skin. J Biomed Opt. 2012; 17:909011.

42. Jacques SL. Optical properties of biological tissues: a review. Phys Med Biol. 2013; 58:R37-R61. [PubMed: 23666068]

43. Rubel DM, et al. Daylight photodynamic therapy with methyl aminolevulinate cream as a convenient, similarly effective, nearly painless alternative to conventional photodynamic therapy in actinic keratosis treatment: a randomized controlled trial. Br J Dermatol. 2014; 171:1164-1171. [PubMed: 24861492]

44. Willey A, Anderson RR, Sakamoto FH. Temperature-Modulated Photodynamic Therapy for the Treatment of Actinic Keratosis on the Extremities. Dermatologic Surg. 2014; 40:1094-1102. 
45. Mamalis A, Koo E, Sckisel G, Siegel D, Jagdeo J. Temperature-dependent impact of thermal aminolaevulinicacid photodynamic therapy on apoptosis and reactiveoxygen species generation in human dermal fibroblasts. Br J Dermatol. 2016; 175:458-459. [PubMed: 27632962] 


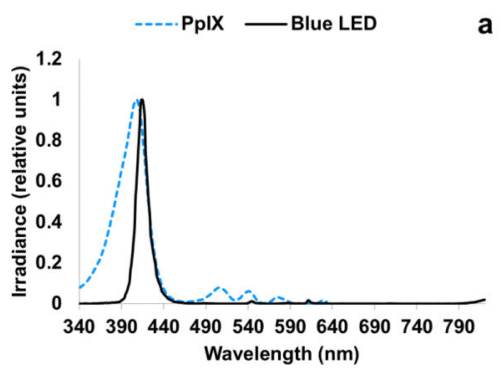

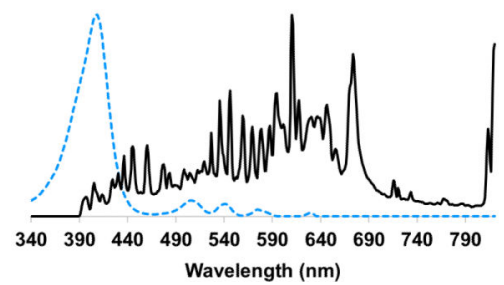

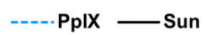

C

Figure 1.

Emission spectra of the three light sources used in this study, overlaid with the absorption spectrum of PpIX. a) $415 \mathrm{~nm}$ LED, b) ceramic metal halide, c) natural sunlight. Each spectrum is normalized to its respective peak maximum for visualization. 

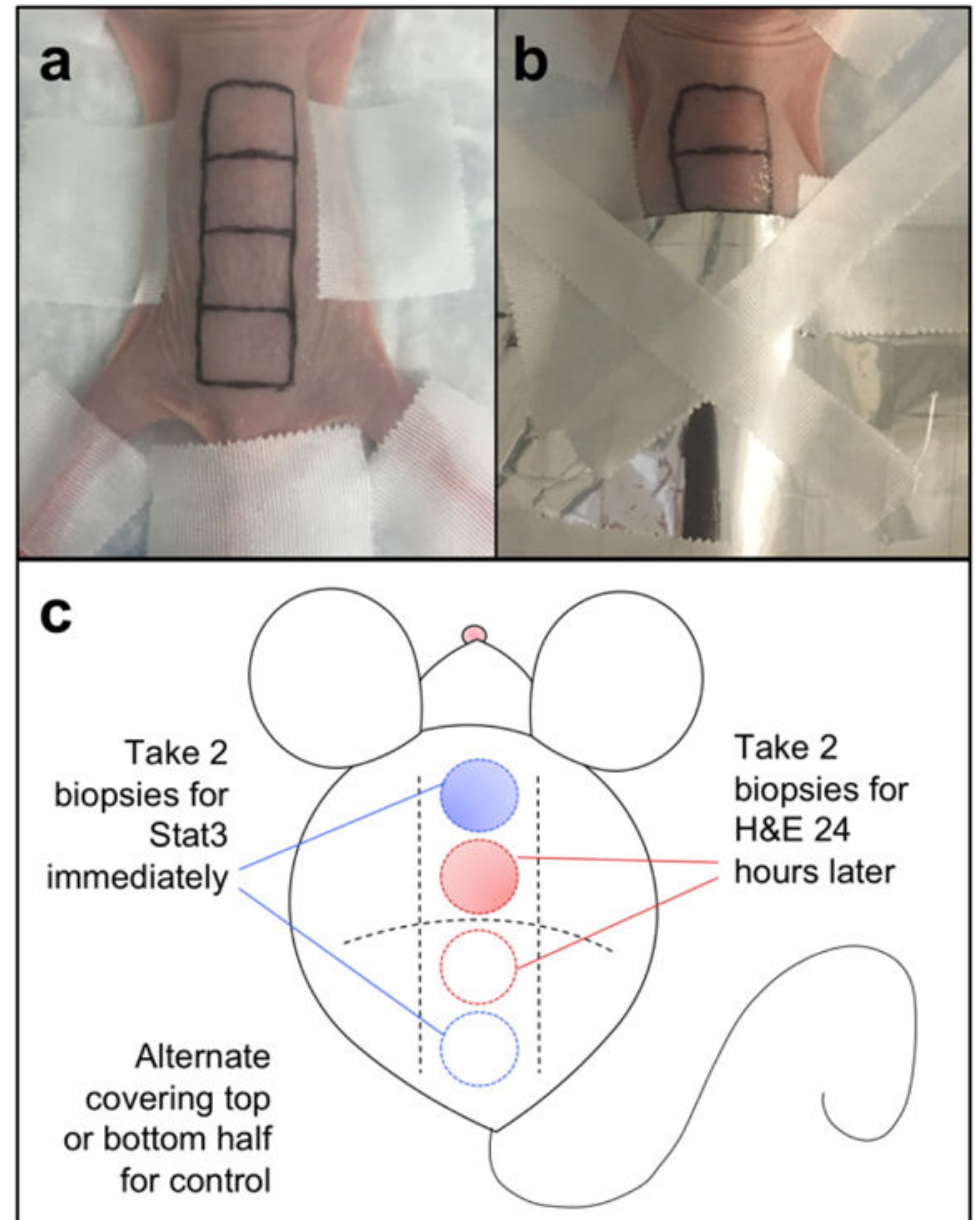

Figure 2.

a) Layout of the treatment field. b) Light-impermeable dressing used to cover the control field (control $=+$ photosensitizer, -light). c) Schematic of post-treatment biopsies to be taken for analysis. 


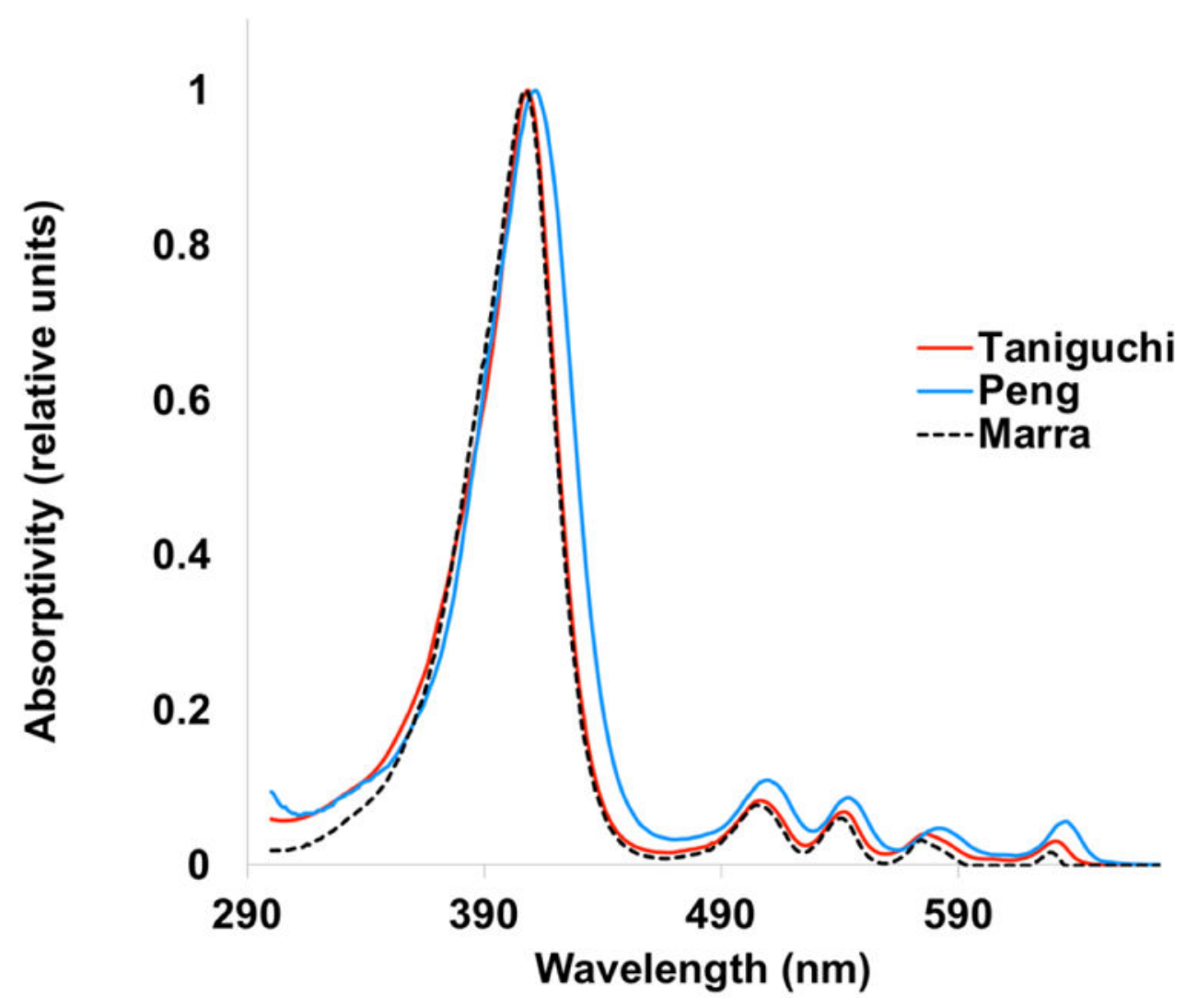

Figure 3.

Various absorption spectra of PpIX in solution. Small shifts due to solvent and molar concentration are observed. Data has been adapted and displayed here with permissions from the authors and publishers. 


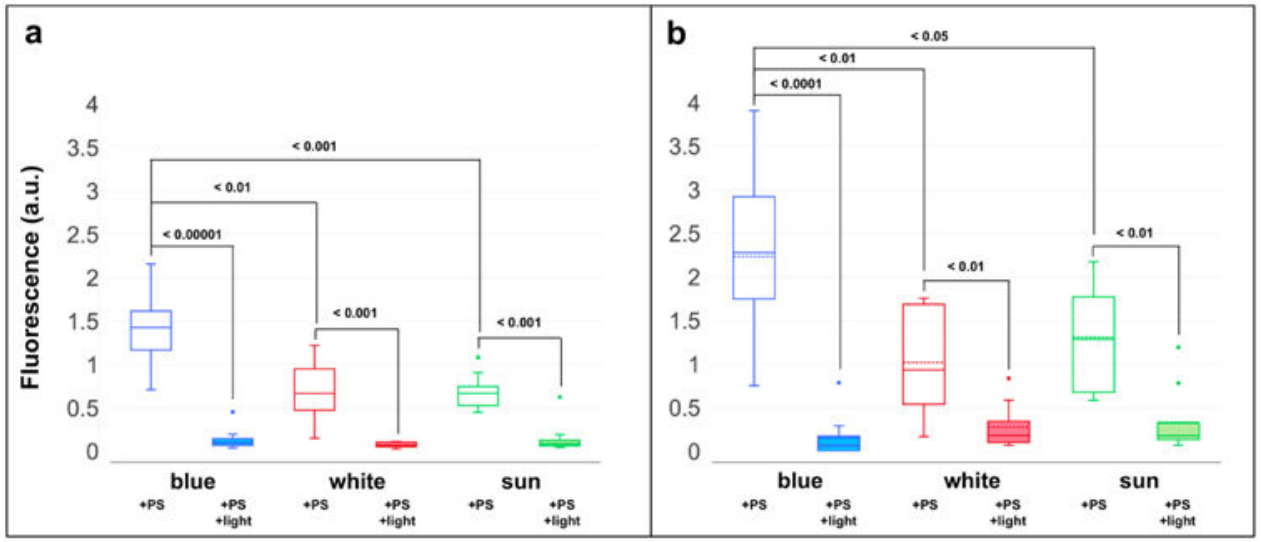

Figure 4.

Each light source is an effective mediator of photobleaching PpIX. a) PpIX is detected by blue laser excitation, corresponding to shallow depths in the skin. b) A red laser was used to probe deeper levels of PpIX. +PS indicates region received photosensitizer. 

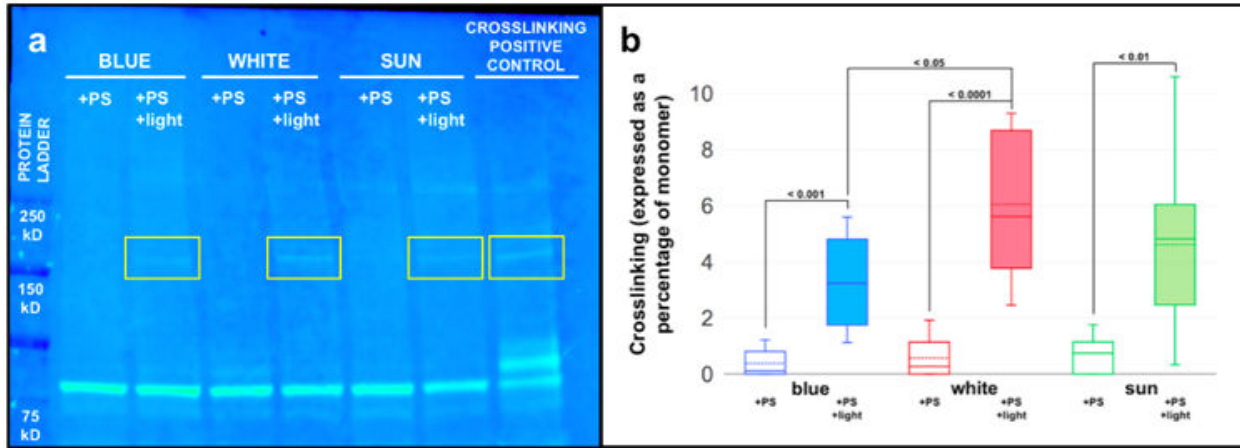

Figure 5.

Stat 3 crosslinking is detectable in each light group. a) A representative immunoblot showing homodimeric Stat3 complexes outlined in yellow. A positive control (high dose, fractionated PDT) was included. b) Intensity of bands was quantified and normalized to monomer. +PS indicates region received photosensitizer. 


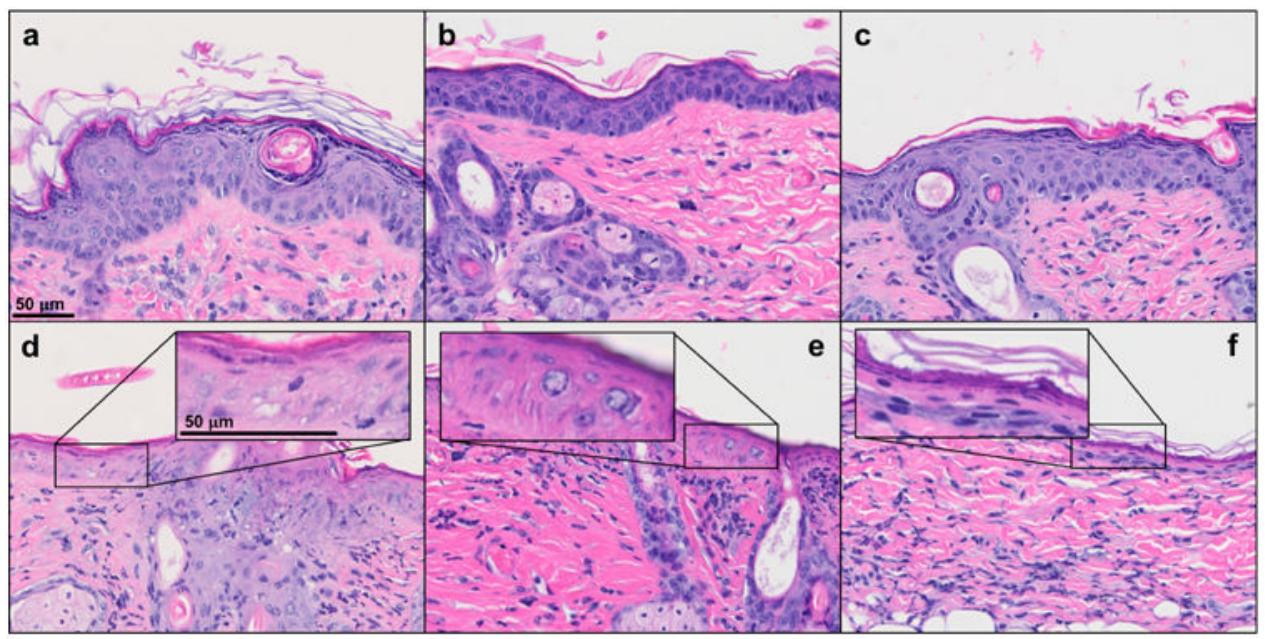

Figure 6.

A representative panel demonstrating that PDT damage is obvious in each light group (d-f), characterized by condensation of nuclear material, hypereosinophilia, and shrinking of epidermal thickness. Control tissue a) blue, b) white, c) sun. Treated tissue d) blue, e) white, f) sun. 


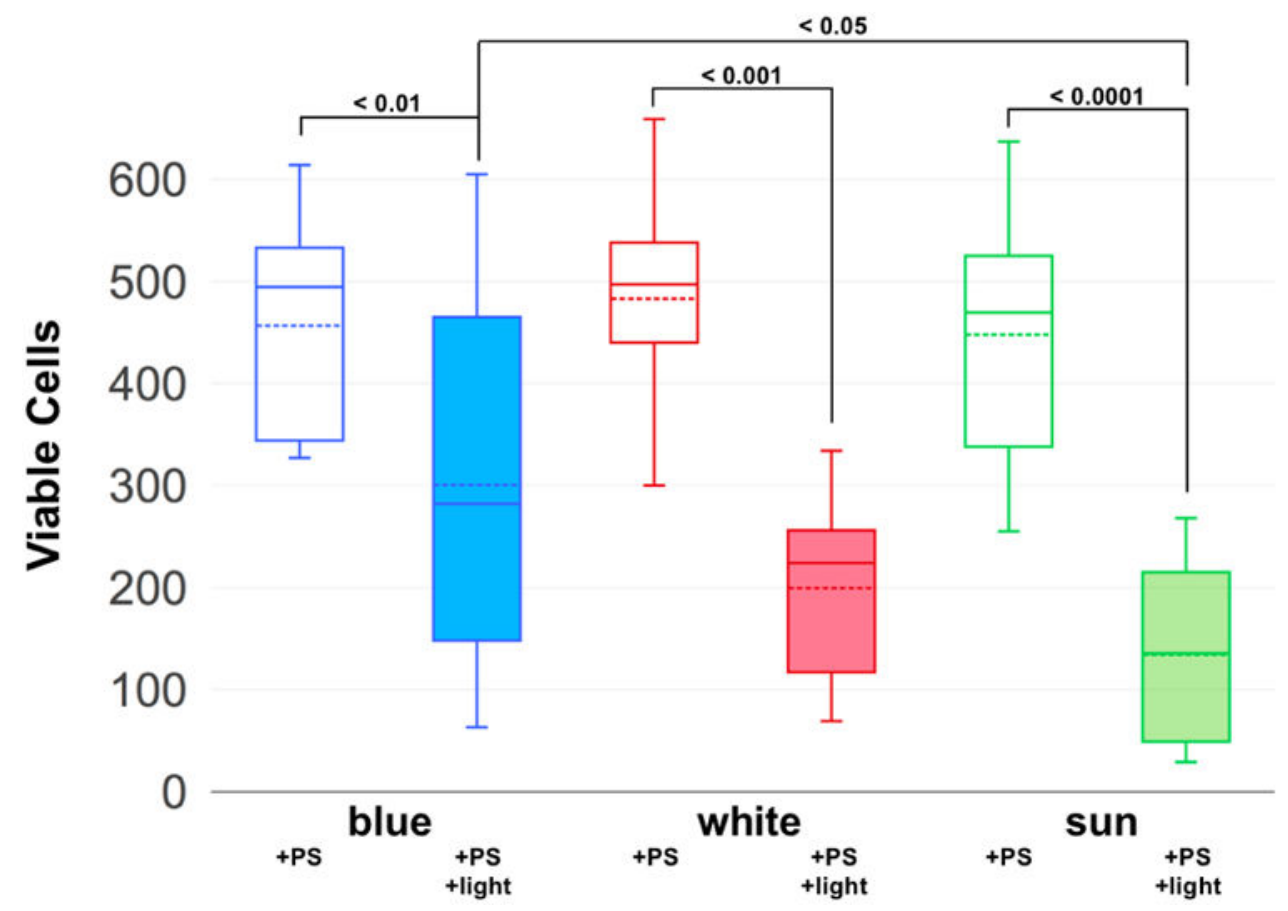

Figure 7.

Healthy nuclei counted in epidermis. Quantification reveals that the blue group causes significantly less damage than the sun group. +PS indicates region received photosensitizer. 


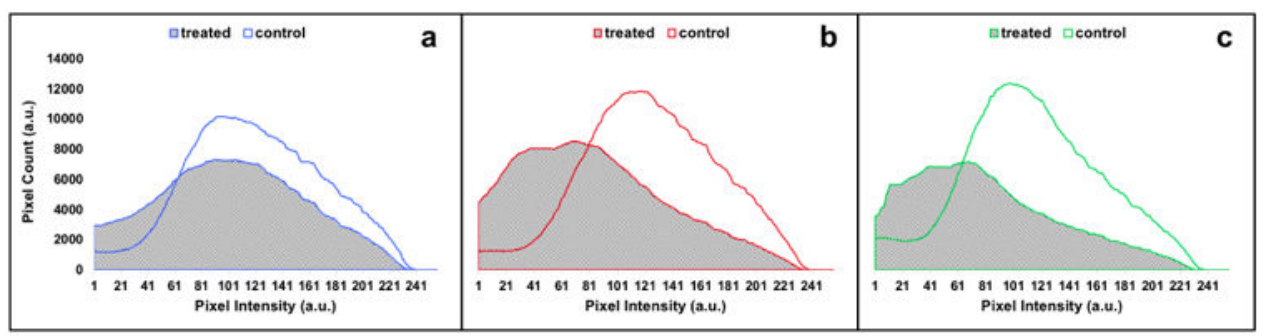

Figure 8.

Epidermal damage can be visualized indirectly through the hematoxylin staining profile. The blue group (a) shows the subtlest change between treated and control, though there is an overall decrease in area. The white (b) and sun (c) groups show a distinct peak shift, indicating a dramatic loss of hematoxylin staining. 


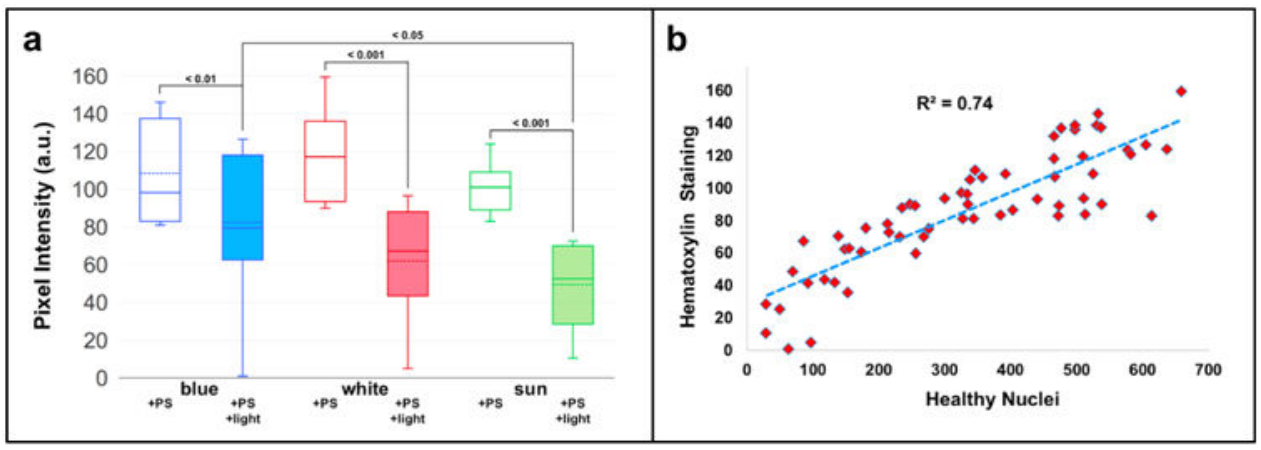

Figure 9.

Statistical analysis of peak maxima (a) reveals almost identical trends to the nuclei counting method (b). +PS indicates region received photosensitizer. 
Table 1

The calculated total light dose is dependent on which PpIX spectrum is used.

\begin{tabular}{|lccc|}
\hline \multicolumn{4}{|c|}{ Variance among PpIX-Weighted Light Doses } \\
\hline Effective Fluence (J/cm $\left.{ }^{2}\right)$ \\
Blue & 20 & 23 & 19 \\
Sun & 20 & 29 & 17 \\
\hline PpIX Spectrum & Taniguchi & Peng & Marra \\
\hline
\end{tabular}




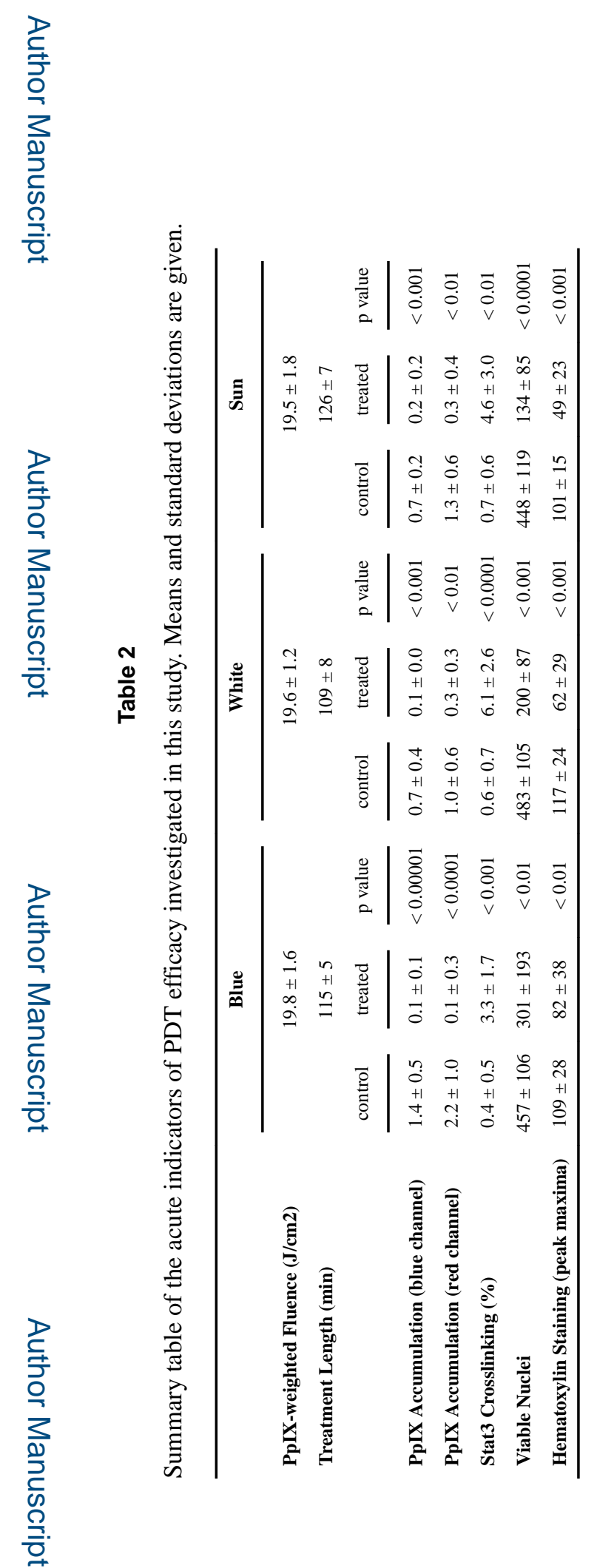

Photochem Photobiol. Author manuscript; available in PMC 2019 September 01. 